\title{
High-order splitting schemes for semilinear evolution equations
}

\author{
Eskil Hansen · Alexander Ostermann
}

\begin{abstract}
We first derive necessary and sufficient stiff order conditions, up to order four, for exponential splitting schemes applied to semilinear evolution equations. The main idea is to identify the local splitting error as a sum of quadrature errors. The order conditions of the quadrature rules then yield the stiff order conditions in an explicit fashion, similarly to that of Runge-Kutta schemes. Furthermore, the derived stiff conditions coincide with the classical non-stiff conditions. Secondly, we propose an abstract convergence analysis, where the linear part of the vector field is assumed to generate a group or a semigroup and the nonlinear part is assumed to be smooth and to satisfy a set of compatibility requirements. Concrete applications include nonlinear wave equations and diffusion-reaction processes. The convergence analysis also extends to the case where the nonlinear flows in the exponential splitting scheme are approximated by a sufficiently accurate one-step method.
\end{abstract}

Keywords Splitting schemes - Exponential splitting - Semilinear evolution equations · High-order methods · Stiff orders · Convergence

Mathematics Subject Classification (2000) $65 \mathrm{~J} 08 \cdot 65 \mathrm{~L} 04 \cdot 65 \mathrm{M} 12 \cdot 65 \mathrm{M} 15$

\section{Introduction}

Semilinear evolution equations

$$
\dot{u}=A u+g(u), \quad u(0)=u_{0},
$$

The work of the first author was supported by the Swedish Research Council under grant 621-2011-5588.

\section{Eskil Hansen}

Centre for Mathematical Sciences, Lund University, P.O. Box 118, SE-221 00 Lund, Sweden

E-mail: eskil@maths.lth.se

Alexander Ostermann

Institut für Mathematik, Universität Innsbruck,Technikerstraße 13, A-6020 Innsbruck, Austria

E-mail: alexander.ostermann@uibk.ac.at 
are frequently encountered in biology, chemistry and physics as this equation type includes nonlinear wave propagation, diffusion-reaction processes and the Schrödinger equation with nonlinear potentials. Here, $A$ is a linear unbounded operator that generates a semigroup $\mathrm{e}^{t A}$ and the nonlinear map $g$ is assumed to be sufficiently many times differentiable. The actions of the nonlinear flow $\psi_{t}$, generated by $g$, and the linear flow $\mathrm{e}^{t A}$ can commonly be computed in a much more efficient manner compared to the flow of the full vector field $A+g$. Hence, an exponential splitting scheme, where $S^{n} u_{0} \approx u(n \tau)$ and

$$
S=\mathrm{e}^{\alpha_{q} \tau A} \psi_{\beta_{q-1} \tau} \mathrm{e}^{\alpha_{q-1} \tau A} \ldots \psi_{\beta_{1} \tau} \mathrm{e}^{\alpha_{1} \tau A},
$$

is often a very efficient choice of temporal discretization. For an introductory reading on splitting schemes we refer to $[6,11]$.

As a prototypical example consider the approximation of the nonlinear wave equation

$$
v_{t t}+v_{t}=c \Delta v+f(v)
$$

which arises, e.g., in physics with $f=$ sin when modeling the dynamics of a Josephson junction [18, Chapter IV.2]. Rewriting it as a first-order system yields a semilinear evolution equation (1.1) governed by the vector field

$$
A u+g(u)=\left(\begin{array}{cc}
0 & I \\
c \Delta & -I
\end{array}\right) u+\left(\begin{array}{c}
0 \\
f(v)
\end{array}\right)
$$

where $u=\left(v, v_{t}\right)^{\mathrm{T}}$. In the context of periodic boundary conditions the action of the linear flow $\mathrm{e}^{t A}$ can be efficiently approximated by a FFT-based scheme. The nonlinear flow has the trivial form

$$
\psi_{t}\left(\begin{array}{c}
v \\
w
\end{array}\right)=\left(\begin{array}{c}
v \\
w+t f(v)
\end{array}\right) .
$$

As seen from Figure 1.1, the non-stiff convergence orders of the second-order Strang splitting, where

$$
S=\mathrm{e}^{\frac{1}{2} \tau A} \psi_{\tau} \mathrm{e}^{\frac{1}{2} \tau A},
$$

and the fourth-order Suzuki scheme (5.2) indeed show up in this infinite dimensional setting of the nonlinear wave equation.

Error analyses for the Strang splitting have been derived in a variety of linear and semilinear settings, see for example $[1,4,7,10,12,13]$. That high-order splitting schemes retain their non-stiff orders of convergence when applied to linear evolution equations has been proven in $[8,19]$ under various regularity and compatibility requirements. It should also be noted that splitting schemes typically suffer from order reductions if the nonlinear flow does not preserve regularity and boundary conditions. Non-stiff order conditions for composition methods, which include exponential splitting schemes, applied to nonlinear ODEs have been derived in [14]; see also [5, Section 2] and [6, Section III.5.3]. However, stiff order conditions and a convergence analysis for high-order exponential splitting schemes applied to semilinear evolution equations is still lacking in the literature.

Hence, the purpose of this study is twofold: first, we aim to derive necessary and sufficient stiff order conditions in the semilinear context, as done up to order four 

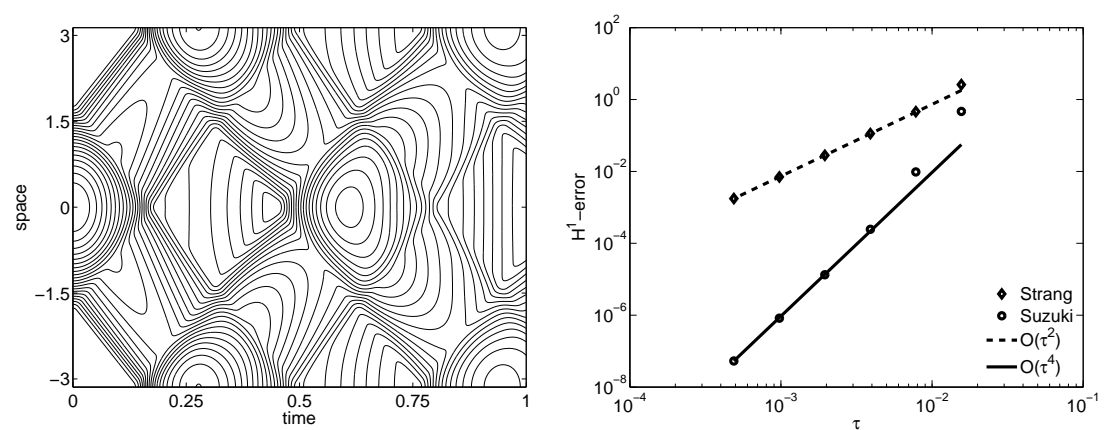

Fig. 1.1 The approximate solution $v$ [left] of the nonlinear wave equation (1.3), and the global $H^{1}$-errors of the full system at time $t=1$ with respect to various time steps $\tau$ [right] of the Strang (1.4) and the Suzuki (5.2) splitting, respectively. Note that the non-stiff convergence orders of two and four, respectively, are present. Here, periodic boundary conditions are imposed, $v(0)$ is smooth, $v_{t}(0)=0, c=100, f=$ $200 \cdot \sin$. The equation is discretized in space by the pseudo-spectral method with $2^{10}$ nodes, and the reference solution is computed by the Suzuki splitting with $\tau=2^{-13}$.

in Sections 3-5. The main idea is in the sprit of [12, Theorem 2.1], namely to identify the local errors of the splitting schemes as a sum of quadrature errors. Secondly, we seek a framework which enables a convergence analysis for exponential splitting schemes in the semilinear and infinite dimensional setting at hand. This is done in Section 6, by utilizing the linear semigroup theory [15]. These considerations also yield convergence of splitting schemes for which the nonlinear flows are approximated by one-step methods, as proven in Section 7 .

\section{Quadrature representations of the local error}

Consider an exponential splitting scheme $S$ of the form (1.2) which satisfies the consistency condition

$$
\sum_{i=1}^{q} \alpha_{i}=1 .
$$

As the stiffness of the evolution equation is assumed to be caused by the operator $A$, sufficient conditions for stiff order $p$ are obtained if the local error $u(\tau)-S u_{0}$ has a representation consisting of (finitely many) $\tau^{p+1}$ terms, each with at most $p$ compositions of $A$.

One way to derive such a local error representation is as follows: first, expand the solution at $t=\tau$ by starting from the variation-of-constants formula

$$
u(\tau)=\mathrm{e}^{\tau A} u_{0}+\int_{0}^{\tau} \mathrm{e}^{(\tau-s) A} g(u(s)) \mathrm{d} s .
$$

Then recursively apply this formula and expand the $g$ terms around the linear flow $\mathrm{e}^{\tau A} u_{0}$. This yields a representation of the form

$$
u(\tau)=\mathrm{e}^{\tau A} u_{0}+\sum_{\ell=1}^{p} I_{\ell} u_{0}+R_{p+1}^{I},
$$


where $I_{\ell} v$ is a linear combination of integral terms with their domain of integrations in $(0, \tau)^{\ell}$. The integrands only contain terms composed of $\mathrm{e}^{t A}$ and the first $(\ell-1)$ derivatives of the nonlinear map $g$, all applied to the vector $v$. The rest term $R_{p+1}^{I}$ has a similar structure with a domain of integration in $(0, \tau)^{p+1}$ and derivatives of $g$ up to order $p$, but also depends on the exact solution $u(t)$ for $t \in[0, \tau]$.

Secondly, rewrite the numerical approximation $S u_{0}$ by only expanding the nonlinear flows $\psi_{\beta_{i} \tau}$ at $t=0$. This yields that

$$
S u_{0}=\mathrm{e}^{\tau A} u_{0}+\sum_{\ell=1}^{p} \tau^{\ell} Q_{\ell} u_{0}+\tau^{p+1} R_{p+1}^{Q},
$$

where $Q_{\ell}$ is again a linear combination of terms composed by e $\mathrm{e}^{t A}$ and the first $(\ell-$ 1) derivatives of $g$. The term $R_{p+1}^{Q}$ has the same structure, apart from containing compositions with $\psi_{t}$. Finally, the local error can be formulated as

$$
u(\tau)-S u_{0}=\sum_{\ell=1}^{p}\left(I_{\ell}-\tau^{\ell} Q_{\ell}\right) u_{0}+\tau^{p+1} R_{p+1}^{R} .
$$

By the above representation and the fact that the error of a quadrature rule of order $\ell$ can be expressed in terms of the $\ell$ th derivative of the integrand, we obtain the following sufficient characterization of the stiff order:

Lemma 2.1 If the terms $Q_{\ell}$ given by an exponential splitting scheme $S$ give rise to quadrature rules of order $(p-\ell+1)$, i.e.,

$$
\left(I_{\ell}-\tau^{\ell} Q_{\ell}\right) u_{0}=\tau^{p+1} R_{\ell} \quad \text { for } \ell=1, \ldots, p
$$

with a bounded rest term $R_{\ell}$, then $S$ has a stiff order of $p$.

\section{Strang splitting}

Before we investigate the stiff order conditions for high-order schemes, we illustrate our approach and introduce some notation by explicitly deriving the quadrature representation of the local error for the Strang splitting (1.4). As stated in the introduction, the Strang splitting is already known to have a stiff order of $p=2$ for semilinear equations. From now on we employ the short-hand notation

$$
\tilde{g}_{s}^{(k)}=g^{(k)}\left(\mathrm{e}^{s A} u_{0}\right) \quad \text { and } \quad g_{1, \ldots, i}^{(k)}=g^{(k)}\left(\mathrm{e}^{\left(\alpha_{1}+\ldots+\alpha_{i}\right) \tau A} u_{0}\right) .
$$

The second-order expansion of the analytical solution is then given by

$$
u(\tau)=\mathrm{e}^{\tau A} u_{0}+\int_{0}^{\tau} \mathrm{e}^{(\tau-s) A} \tilde{g}_{s} \mathrm{~d} s+\int_{0}^{\tau} \int_{0}^{s} \mathrm{e}^{(\tau-s) A} \tilde{g}_{s}^{\prime} \mathrm{e}^{(s-\xi) A} \tilde{g}_{\xi} \mathrm{d} \xi \mathrm{d} s+R_{3}^{I},
$$

where the third-order integral is

$$
\begin{aligned}
R_{3}^{I}= & \int_{0}^{\tau} \mathrm{e}^{(\tau-s) A} \int_{0}^{s} \int_{0}^{1}\left(\int_{0}^{\xi} \tilde{g}_{s}^{\prime} \mathrm{e}^{(s-\xi) A} g^{\prime}\left(v_{\xi}(\eta)\right) \mathrm{e}^{(\xi-\gamma) A} g(u(\gamma)) \mathrm{d} \gamma\right. \\
& \left.+\int_{0}^{s}(1-\eta) g^{\prime \prime}\left(v_{s}(\eta)\right)\left[\mathrm{e}^{(s-\xi) A} g(u(\xi)), \mathrm{e}^{(s-\gamma) A} g(u(\gamma))\right] \mathrm{d} \gamma\right) \mathrm{d} \eta \mathrm{d} \xi \mathrm{d} s
\end{aligned}
$$


with $v_{s}(\eta)=\mathrm{e}^{s A} u_{0}+\eta \int_{0}^{s} \mathrm{e}^{(s-\gamma) A} g(u(\gamma)) \mathrm{d} \gamma$. The expansion of the Strang approximation becomes

$$
S u_{0}=\mathrm{e}^{\tau A} u_{0}+\tau \mathrm{e}^{\frac{1}{2} \tau A} g_{1}+\tau^{2} \frac{1}{2} \mathrm{e}^{\frac{1}{2} \tau A} g_{1}^{\prime} g_{1}+\tau^{3} R_{3}^{Q}
$$

with the rest term

$R_{3}^{Q}=\frac{1}{2} \mathrm{e}^{\frac{1}{2} \tau A} \int_{0}^{1}(1-\eta)^{2}\left(g^{\prime}(w(\eta))^{2} g(w(\eta))+g^{\prime \prime}(w(\eta))[g(w(\eta)), g(w(\eta))]\right) \mathrm{d} \eta$,

where $w(\eta)=\psi_{\eta \tau}\left(\mathrm{e}^{\frac{1}{2} \tau A} u_{0}\right)$. With this in place, one directly obtains that the first quadrature rule $Q_{1}$ is nothing else but the second-order midpoint rule, i.e.,

$$
\left(I_{1}-\tau Q_{1}\right) u_{0}=\int_{0}^{\tau} \mathrm{e}^{(\tau-s) A} g\left(\mathrm{e}^{s A} u_{0}\right) \mathrm{d} s-\tau \mathrm{e}^{\frac{1}{2} \tau A} g\left(\mathrm{e}^{\frac{1}{2} \tau A} u_{0}\right)=\tau^{3} R_{1},
$$

and $R_{1}$ contains compositions of at most two $A$ terms. The second quadrature rule $Q_{2}$ can be identified as a first-order approximation of the double integral $I_{2}$ over a domain in $(0, \tau)^{2}$, which yields that

$$
\left(I_{2}-\tau^{2} Q_{2}\right) u_{0}=\tau^{3} R_{2},
$$

where $R_{2}$ contains at most a single $A$ term. Hence, the Strang splitting has a stiff order of $p=2$.

\section{Third-order schemes}

We continue by deriving the stiff order conditions for exponential splitting schemes with $q=3$, i.e.,

$$
S=\mathrm{e}^{\alpha_{3} \tau A} \psi_{\beta_{2} \tau} \mathrm{e}^{\alpha_{2} \tau A} \psi_{\beta_{1} \tau} \mathrm{e}^{\alpha_{1} \tau A} .
$$

A concrete example of such a third-order scheme [9,17], with non-stiff order $p=3$, is given by the parameters

$$
\begin{gathered}
\alpha_{1}=\frac{1}{4}+\mathrm{i} \frac{\sqrt{3}}{12}, \quad \alpha_{2}=\frac{1}{2}, \quad \alpha_{3}=\frac{1}{4}-\mathrm{i} \frac{\sqrt{3}}{12}, \\
\beta_{1}=\frac{1}{2}+\mathrm{i} \frac{\sqrt{3}}{6}, \quad \text { and } \quad \beta_{2}=\frac{1}{2}-\mathrm{i} \frac{\sqrt{3}}{6} .
\end{gathered}
$$

The third-order expansion of the analytical solution

$$
u(\tau)=\mathrm{e}^{\tau A} u_{0}+\left(I_{1}+I_{2}+I_{3}^{(a)}+I_{3}^{(b)}\right) u_{0}+R_{4}^{I}
$$

is then given by the integrals

$$
\begin{aligned}
I_{1} u_{0} & =\int_{0}^{\tau} \mathrm{e}^{(\tau-s) A} \tilde{g}_{s} \mathrm{~d} s \\
I_{2} u_{0} & =\int_{0}^{\tau} \int_{0}^{s} \mathrm{e}^{(\tau-s) A} \tilde{g}_{s}^{\prime} \mathrm{e}^{(s-\xi) A} \tilde{g}_{\xi} \mathrm{d} \xi \mathrm{d} s, \\
I_{3}^{(a)} u_{0} & =\int_{0}^{\tau} \int_{0}^{s} \int_{0}^{\xi} \mathrm{e}^{(\tau-s) A} \tilde{g}_{s}^{\prime} \mathrm{e}^{(s-\xi) A} \tilde{g}_{\xi}^{\prime} \mathrm{e}^{(\xi-\sigma) A} \tilde{g}_{\sigma} \mathrm{d} \sigma \mathrm{d} \xi \mathrm{d} s, \\
I_{3}^{(b)} u_{0} & =\frac{1}{2} \int_{0}^{\tau} \int_{0}^{s} \int_{0}^{s} \mathrm{e}^{(\tau-s) A} \tilde{g}_{s}^{\prime \prime}\left[\mathrm{e}^{(s-\xi) A} \tilde{g}_{\xi}, \mathrm{e}^{(s-\sigma) A} \tilde{g}_{\sigma}\right] \mathrm{d} \sigma \mathrm{d} \xi \mathrm{d} s .
\end{aligned}
$$


Note that for $A=0$ the integrands of the above integrals correspond to the rooted trees

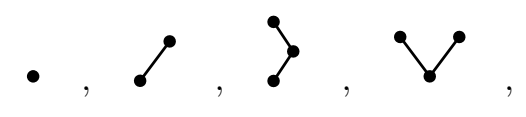

respectively. Furthermore, the third-order expansion of the splitting scheme has the form

$$
S u_{0}=\mathrm{e}^{\tau A} u_{0}+\left(\tau Q_{1}+\tau^{2} Q_{2}+\tau^{3} Q_{3}^{(a)}+\tau^{3} Q_{3}^{(b)}\right) u_{0}+\tau^{4} R_{4}^{Q},
$$

where the quadrature terms are

$$
\begin{aligned}
Q_{1} u_{0}= & \beta_{1} \mathrm{e}^{\left(\alpha_{2}+\alpha_{3}\right) \tau A} g_{1}+\beta_{2} \mathrm{e}^{\alpha_{3} \tau A} g_{1,2}, \\
Q_{2} u_{0}= & \frac{1}{2} \beta_{1}^{2} \mathrm{e}^{\left(\alpha_{2}+\alpha_{3}\right) \tau A} g_{1}^{\prime} g_{1}+\beta_{1} \beta_{2} \mathrm{e}^{\alpha_{3} \tau A} g_{1,2}^{\prime} \mathrm{e}^{\alpha_{2} \tau A} g_{1}+\frac{1}{2} \beta_{2}^{2} \mathrm{e}^{\alpha_{3} \tau A} g_{1,2}^{\prime} g_{1,2}, \\
Q_{3}^{(a)} u_{0}= & \frac{1}{6} \beta_{1}^{3} \mathrm{e}^{\left(\alpha_{2}+\alpha_{3}\right) \tau A} g_{1}^{\prime} g_{1}^{\prime} g_{1}+\frac{1}{2} \beta_{1}^{2} \beta_{2} \mathrm{e}^{\alpha_{3} \tau A} g_{1,2}^{\prime} \mathrm{e}^{\alpha_{2} \tau A} g_{1}^{\prime} g_{1} \\
& +\frac{1}{2} \beta_{1} \beta_{2}^{2} \mathrm{e}^{\alpha_{3} \tau A} g_{1,2}^{\prime} g_{1,2}^{\prime} \mathrm{e}^{\alpha_{2} \tau A} g_{1}+\frac{1}{6} \beta_{2}^{3} \mathrm{e}^{\alpha_{3} \tau A} g_{1,2}^{\prime} g_{1,2}^{\prime} g_{1,2}, \\
Q_{3}^{(b)} u_{0}= & \frac{1}{6} \beta_{1}^{3} \mathrm{e}^{\left(\alpha_{2}+\alpha_{3}\right) \tau A} g_{1}^{\prime \prime}\left[g_{1}, g_{1}\right]+\frac{1}{2} \beta_{1}^{2} \beta_{2} \mathrm{e}^{\alpha_{3} \tau A} g_{1,2}^{\prime \prime}\left[\mathrm{e}^{\alpha_{2} \tau A} g_{1}, \mathrm{e}^{\alpha_{2} \tau A} g_{1}\right] \\
& +\frac{1}{2} \beta_{1} \beta_{2}^{2} \mathrm{e}^{\alpha_{3} \tau A} g_{1,2}^{\prime \prime}\left[\mathrm{e}^{\alpha_{2} \tau A} g_{1}, g_{1,2}\right]+\frac{1}{6} \beta_{2}^{3} \mathrm{e}^{\alpha_{3} \tau A} g_{1,2}^{\prime \prime}\left[g_{1,2}, g_{1,2}\right] .
\end{aligned}
$$

Here, the $Q_{3}^{(a)}$ and $Q_{3}^{(b)}$ terms are identified by their sequences of $g$ derivatives which should match the integrands of $I_{3}^{(a)}$ and $I_{3}^{(b)}$, respectively. That is, case $(a)$ has the pattern $g^{\prime} g^{\prime} g$ and case $(b)$ has $g^{\prime \prime}[g, g]$.

The ansatz that $Q_{\ell}$ gives rise to quadrature rules of order $(p-\ell+1)$ allows us to derive the conditions for the parameters $\left\{\alpha_{i}, \beta_{i}\right\}$ via the classical quadrature order conditions. For example, the expression

$$
Q_{2} u_{0}=\frac{1}{2} \sum_{i=1}^{3} b_{i} f\left(c_{i} \tau, d_{i} \tau\right)
$$

with $f(s, \xi)=\mathrm{e}^{(\tau-s) A} \tilde{g}_{s}^{\prime} \mathrm{e}^{(s-\xi) A} \tilde{g}_{\xi}$ and

$$
\begin{gathered}
b_{1}=\beta_{1}^{2}, \quad b_{2}=2 \beta_{1} \beta_{2}, \quad b_{3}=\beta_{2}^{2}, \\
c_{1}=d_{1}=\alpha_{1}, \quad c_{2}=\alpha_{1}+\alpha_{2}, \quad d_{2}=\alpha_{1}, \quad \text { and } \quad c_{3}=d_{3}=\alpha_{1}+\alpha_{2},
\end{gathered}
$$

is required to be a second-order quadrature rule. Hence, the quadrature parameters need to satisfy the conditions

$$
\sum_{i=1}^{3} b_{i}=1, \quad \sum_{i=1}^{3} b_{i} c_{i}=\frac{2}{3}, \quad \text { and } \quad \sum_{i=1}^{3} b_{i} d_{i}=\frac{1}{3}
$$


This procedure yields the following sufficient conditions for an exponential splitting scheme with $q=3$ to have a stiff order of $p=3$ :

$$
\begin{gathered}
\alpha_{1}+\alpha_{2}+\alpha_{3}=1, \\
\beta_{1}+\beta_{2}=1, \\
\beta_{1} \alpha_{1}+\beta_{2}\left(\alpha_{1}+\alpha_{2}\right)=\frac{1}{2}, \\
\beta_{1} \alpha_{1}^{2}+\beta_{2}\left(\alpha_{1}+\alpha_{2}\right)^{2}=\frac{1}{3}, \\
\beta_{1}^{2} \alpha_{1}+2 \beta_{1} \beta_{2}\left(\alpha_{1}+\alpha_{2}\right)+\beta_{2}^{2}\left(\alpha_{1}+\alpha_{2}\right)=\frac{2}{3}, \\
\beta_{1}^{2} \alpha_{1}+2 \beta_{1} \beta_{2} \alpha_{1}+\beta_{2}^{2}\left(\alpha_{1}+\alpha_{2}\right)=\frac{1}{3} .
\end{gathered}
$$

Note that not all the order conditions stated in (4.2) are necessary. For example, under the first five conditions the sixth follows. This can be proven by adding condition five to six, which reduces to the third condition. The remaining five conditions are also necessary as they are equivalent with the classical non-stiff third-order conditions; see [5, Section 2].

\section{Fourth-order schemes}

Sufficient conditions for an exponential splitting scheme with $q=4$ to have a stiff order of $p=4$ can be derived along the same lines as done in the previous section. One then obtains an additional term $I_{4} u_{0}$ in the expansion of the exact solution, where the four integrands correspond to the rooted trees

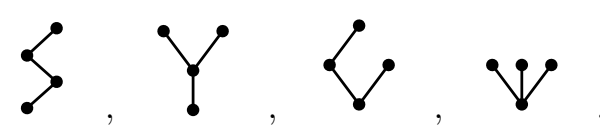

Apart from a rather lengthy expansion of the splitting scheme, the only difficulty with the fourth-order analysis is that one needs to take more care when making the ansatz regarding the forms of the quadrature rules. For example, the quadrature term approximating $I_{3}^{(b)}$ in (4.1) needs to be handled in a symmetric fashion in order not to obtain conditions that contradict those arising from the approximation of $I_{3}^{(a)}$. More precisely, all expressions of the form $g^{\prime \prime}(u)[v, w]$ need to be written as

$$
\frac{1}{2}\left(g^{\prime \prime}(u)[v, w]+g^{\prime \prime}(u)[w, v]\right),
$$

which is possible as $g^{\prime \prime}(u)$ is symmetric, and a symmetric ansatz of the form

$$
\frac{1}{12} \sum_{i=1}^{10} b_{i} f\left(c_{i} \tau, d_{i} \tau, e_{i} \tau\right)+b_{i} f\left(c_{i} \tau, e_{i} \tau, d_{i} \tau\right)
$$


is required, where $f(s, \xi, \sigma)=\mathrm{e}^{(\tau-s) A} \tilde{g}_{s}^{\prime \prime}\left[\mathrm{e}^{(s-\xi) A} \tilde{g}_{\xi}, \mathrm{e}^{(s-\sigma) A} \tilde{g}_{\sigma}\right]$. The sufficient stiff third-order conditions for an exponential splitting scheme with $q=4$ are then

$$
\begin{gathered}
\alpha_{1}+\alpha_{2}+\alpha_{3}+\alpha_{4}=1 \\
\beta_{1}+\beta_{2}+\beta_{3}=1, \\
\beta_{1} \alpha_{1}+\beta_{2}\left(\alpha_{1}+\alpha_{2}\right)+\beta_{3}\left(\alpha_{1}+\alpha_{2}+\alpha_{3}\right)=\frac{1}{2} \\
\beta_{1} \alpha_{1}^{2}+\beta_{2}\left(\alpha_{1}+\alpha_{2}\right)^{2}+\beta_{3}\left(\alpha_{1}+\alpha_{2}+\alpha_{3}\right)^{2}=\frac{1}{3}, \\
\beta_{1}^{2} \alpha_{1}+2 \beta_{1} \beta_{2}\left(\alpha_{1}+\alpha_{2}\right)+\beta_{2}^{2}\left(\alpha_{1}+\alpha_{2}\right)+2 \beta_{1} \beta_{3}\left(\alpha_{1}+\alpha_{2}+\alpha_{3}\right) \\
+2 \beta_{2} \beta_{3}\left(\alpha_{1}+\alpha_{2}+\alpha_{3}\right)+\beta_{3}^{2}\left(\alpha_{1}+\alpha_{2}+\alpha_{3}\right)=\frac{2}{3}, \\
\beta_{1}^{2} \alpha_{1}+2 \beta_{1} \beta_{2} \alpha_{1}+\beta_{2}^{2}\left(\alpha_{1}+\alpha_{2}\right)+2 \beta_{1} \beta_{3} \alpha_{1} \\
+2 \beta_{2} \beta_{3}\left(\alpha_{1}+\alpha_{2}\right)+\beta_{3}^{2}\left(\alpha_{1}+\alpha_{2}+\alpha_{3}\right)=\frac{1}{3}
\end{gathered}
$$

Before we state the sufficient fourth-order conditions, we remark that it is often favorable to allow a few more stages, i.e., choosing a scheme with $q \geq 5$, in order to add more structure to the parameters $\left\{\alpha_{i}, \beta_{i}\right\}$ or to optimize the error constants. A scheme with real parameters of small size, $q=6$, and non-stiff order $p=4$ can be obtained via Suzuki's fractals [17]. The scheme is given by

$$
\begin{gathered}
\alpha_{1}=\alpha_{6}=\frac{1}{8-2 \cdot 4^{1 / 3}}, \quad \alpha_{2}=\alpha_{5}=\frac{1}{4-4^{1 / 3}}, \quad \alpha_{3}=\alpha_{4}=\frac{1-4^{1 / 3}}{8-2 \cdot 4^{1 / 3}}, \quad \text { and } \\
\beta_{1}=\beta_{2}=\beta_{4}=\beta_{5}=\frac{1}{4-4^{1 / 3}}, \quad \beta_{3}=-\frac{4^{1 / 3}}{4-4^{1 / 3}} .
\end{gathered}
$$

For semilinear parabolic equations it is desirable to have the parameters $\alpha_{i}$ in a sector in the right complex half-plane and real parameters $\beta_{i}$, as $\mathrm{e}^{t A}$ is an analytic semigroup and $\psi_{t}$ does not need to be well defined for complex times. The scheme below [3], with $q=5$ and a non-stiff order of $p=4$, fits into this context.

$$
\begin{gathered}
\alpha_{1}=\alpha_{5}=\frac{1}{10}-\mathrm{i} \frac{1}{30}, \quad \alpha_{2}=\alpha_{4}=\frac{4}{15}+\mathrm{i} \frac{2}{15}, \quad \alpha_{3}=\frac{4}{15}-\mathrm{i} \frac{1}{5}, \quad \text { and } \\
\beta_{1}=\beta_{2}=\beta_{3}=\beta_{4}=\frac{1}{4} .
\end{gathered}
$$

Schemes with optimized error constants can, e.g., be found in [2].

Hence, we would also like to have the sufficient stiff fourth-order conditions for an exponential splitting scheme $S$ with an arbitrary number of stages. By observing how the first three quadrature terms $Q_{\ell}$ change when passing from $q=3$ to $q=4$, compare (4.2) with (5.1), it is straightforward to derive the general structure of the fourth-order quadrature conditions for any $q \geq 4$. These sufficient conditions are stated in Table 5.1. As for the third-order schemes in Section 4, the identification of the local splitting error as a sum of quadrature errors yields redundant stiff order conditions. In fact, under the conditions stated in Table 5.1, only the eight conditions $\{\mathrm{c}(i, j)\}$ are necessary; the remaining five conditions $\{\mathrm{r}(i, j)\}$ are redundant. For example, $\mathrm{c}(1,1)$ allows us to express

$$
\sum_{j=1}^{i-1} \beta_{j}=1-\sum_{j=i}^{q-1} \beta_{j} .
$$


Table 5.1 Sufficient conditions for a splitting scheme of the form $S=\mathrm{e}^{\alpha_{q} \tau A} \psi_{\beta_{q-1} \tau} \mathrm{e}^{\alpha_{q-1} \tau A} \ldots \psi_{\beta_{1} \tau} \mathrm{e}^{\alpha_{1} \tau A}$ to have a stiff order of $p \leq 4$. The $j$ th condition of order $i$ has the name $\mathrm{c}(i, j)$ or $\mathrm{r}(i, j)$ depending on whether the condition is necessary (c) or redundant (r).

\begin{tabular}{|c|c|}
\hline \multicolumn{2}{|r|}{ Conditions } \\
\hline $\mathrm{c}(0,1)$ & $\alpha_{1}+\ldots+\alpha_{q}=1$ \\
\hline $\mathrm{c}(1,1)$ & $\beta_{1}+\ldots+\beta_{q-1}=1$ \\
\hline $\mathrm{c}(2,1)$ & $\beta_{1} c_{1}+\beta_{2} c_{2}+\ldots+\beta_{q-1} c_{q-1}=1 / 2$ \\
\hline $\mathrm{c}(3,1)$ & $\beta_{1} c_{1}^{2}+\beta_{2} c_{2}^{2}+\ldots+\beta_{q-1} c_{q-1}^{2}=1 / 3$ \\
\hline \multirow{2}{*}{$\begin{array}{l}\mathrm{c}(3,2) \\
\mathrm{r}(3,3)\end{array}$} & $2 \sum_{i=1}^{q-1} \sum_{j=1}^{i-1} \beta_{i} \beta_{j} c_{i}+\sum_{i=1}^{q-1} \beta_{i}^{2} c_{i}=2 / 3$ \\
\hline & $2 \sum_{i=1}^{q-1} \sum_{j=1}^{i-1} \beta_{i} \beta_{j} c_{j}+\sum_{i=1}^{q-1} \beta_{i}^{2} c_{i}=1 / 3$ \\
\hline $\mathrm{c}(4,1)$ & $\beta_{1} c_{1}^{3}+\beta_{2} c_{2}^{3}+\ldots+\beta_{q-1} c_{q-1}^{3}=1 / 4$ \\
\hline$c(4,2)$ & $2 \sum_{i=1}^{q-1} \sum_{j=1}^{i-1} \beta_{i} \beta_{j} c_{i}^{2}+\sum_{i=1}^{q-1} \beta_{i}^{2} c_{i}^{2}=1 / 2$ \\
\hline \multirow[t]{2}{*}{$\mathrm{c}(4,3)$} & $6 \sum_{i=1}^{q-1} \sum_{j=1}^{i-1} \sum_{k=1}^{j-1} \beta_{i} \beta_{j} \beta_{k} c_{i}+3 \sum_{i=1}^{q-1} \sum_{j=1}^{i-1}\left(\beta_{i}^{2} \beta_{j}+\beta_{i} \beta_{j}^{2}\right) c_{i}$ \\
\hline & $+\sum_{i=1}^{q-1} \beta_{i}^{3} c_{i}=3 / 4$ \\
\hline $\mathrm{r}(4,4)$ & $2 \sum_{i=1}^{q-1} \sum_{j=1}^{i-1} \beta_{i} \beta_{j} c_{i} c_{j}+\sum_{i=1}^{q-1} \beta_{i}^{2} c_{i}^{2}=1 / 4$ \\
\hline $\mathrm{r}(4,5)$ & $2 \sum_{i=1}^{q-1} \sum_{j=1}^{i-1} \beta_{i} \beta_{j} c_{j}^{2}+\sum_{i=1}^{q-1} \beta_{i}^{2} c_{i}^{2}=1 / 6$ \\
\hline \multirow[t]{2}{*}{$\mathrm{r}(4,6)$} & $6 \sum_{i=1}^{q-1} \sum_{j=1}^{i-1} \sum_{k=1}^{j-1} \beta_{i} \beta_{j} \beta_{k} c_{j}+3 \sum_{i=1}^{q-1} \sum_{j=1}^{i-1}\left(\beta_{i}^{2} \beta_{j} c_{i}+\beta_{i} \beta_{j}^{2} c_{j}\right)$ \\
\hline & $+\sum_{i=1}^{q-1} \beta_{i}^{3} c_{i}=1 / 2$ \\
\hline \multirow[t]{2}{*}{$\mathrm{r}(4,7)$} & $6 \sum_{i=1}^{q-1} \sum_{j=1}^{i-1} \sum_{k=1}^{j-1} \beta_{i} \beta_{j} \beta_{k} c_{k}+3 \sum_{i=1}^{q-1} \sum_{j=1}^{i-1}\left(\beta_{i}^{2} \beta_{j}+\beta_{i} \beta_{j}^{2}\right) c_{j}$ \\
\hline & $+\sum_{i=1}^{q-1} \beta_{i}^{3} c_{i}=1 / 4$ \\
\hline \multicolumn{2}{|r|}{ Notation } \\
\hline \multicolumn{2}{|r|}{$c_{i}:=\alpha_{1}+\ldots+\alpha_{i}$} \\
\hline
\end{tabular}

Using this in $\mathrm{c}(3,2)$, employing $\mathrm{c}(2,1)$ and interchanging summation eventually yields condition $r(3,3)$. Condition $r(4,4)$ is obtained by squaring $\mathrm{c}(2,1)$. Employing (5.3) in $\mathrm{c}(4,2)$, interchanging summation and using $\mathrm{c}(3,1)$ yields condition $\mathrm{r}(4,5)$. In a similar way, one verifies the redundancy of $\mathrm{r}(4,6)$ and $\mathrm{r}(4,7)$. Furthermore, the conditions $\{\mathrm{c}(i, j)\}$ are also necessary, as they are equivalent to the standard non-stiff order conditions. In conclusion, we obtain the theorem below. 
Theorem 5.1 Let $p \leq 4$ and consider the discretization of the semilinear evolution equation (1.1) by an exponential splitting scheme $S$ given in (1.2). If $S$ has a non-stiff order of $p$ then it also has a stiff order of $p$.

\section{Convergence analysis}

With the stiff order concept in place, the convergence analysis follows by establishing a framework which yields that the quadrature errors $R_{\ell}$ are all bounded, as well as giving rise to a solution and a stability concept. In the context of semilinear PDEs, deriving regularity and stability results for the exact flow and the splitting scheme, respectively, commonly requires a deep analysis tailored to the specific equation. We will therefore restrict our attention to the framework below, where the vector field $g$ is assumed to be sufficiently smooth. Results tailored for specific vector fields with less regularity will be studied elsewhere.

For the sake of notational simplicity, we only consider evolution equations solved forward in time and splitting schemes for which the parameters $\alpha_{i}$ are either real (in the case of a $C_{0}$ group) or lie in the sector $\Sigma_{\phi}=\{z \in \mathbb{C}:|\arg z| \leq \phi\}$, with $\phi \in$ $[0, \pi / 2)$, and the parameters $\beta_{i}$ are real. Let $\mathscr{X}$ be a Banach space with norm $\|\cdot\|$ and let $\mathscr{B}_{r}=\{u \in \mathscr{X}:\|u\| \leq r\}$. The induced operator norm on $\mathscr{X}$ is also denoted by $\|\cdot\|$. We then consider evolution equations of the form (1.1) on $\mathscr{X}$, where the vector field $A+g$ fulfills the properties below.

Assumption 6.1 The linear operator $A: \mathscr{D}(A) \subseteq \mathscr{X} \rightarrow \mathscr{X}$ is closed, densely defined and generates one of the following:

(a) a $C_{0}$ group $\mathrm{e}^{t A}: \mathscr{X} \rightarrow \mathscr{X}$ satisfying the bound $\left\|\mathrm{e}^{t A}\right\| \leq 1$ for all $t \in \mathbb{R}$;

(b) an analytic semigroup $\mathrm{e}^{t A}: \mathscr{X} \rightarrow \mathscr{X}$ defined in an open sector with angle $\phi^{\prime} \in$ $(\phi, \pi / 2]$, and $\left\|\mathrm{e}^{t A}\right\| \leq 1$ for all $t \in \Sigma_{\phi}$

Note that if all $\alpha_{i}$ are nonnegative and $\beta_{i}$ are real, which only occurs for schemes of order $p \leq 2$, then it is sufficient to just assume that $A$ generates a $C_{0}$ semigroup. Furthermore, the assumed bound $\left\|\mathrm{e}^{t A}\right\| \leq 1$ is not restrictive as it is valid for any $C_{0}$ semigroup on $\mathscr{X}$ after rescaling the equation and equipping the space with a suitable equivalent norm.

Assumption 6.2 Consider the Banach space $\mathscr{D}\left(A^{k}\right)$ equipped with the graph norm and define $\mathscr{D}\left(A^{0}\right)=\mathscr{X}$. For a fixed $p \geq 1$ and $k=0, \ldots, p$, the nonlinear operator $g$ maps $\mathscr{D}\left(A^{k}\right)$ into itself and it is locally Lipschitz continuous on $\mathscr{D}\left(A^{p}\right)$. For $\ell=$ $1, \ldots, p$, the operator $g$ is $\ell$-times continuously Fréchet differentiable on $\mathscr{D}\left(A^{k}\right)$ with $k=0, \ldots, p-\ell$.

As $\mathrm{e}^{t A}$ is also a $C_{0}$ semigroup on $\mathscr{D}\left(A^{p}\right)$ and $g$ is locally Lipschitz continuous on the same space, it follows that for every $u_{0} \in \mathscr{D}\left(A^{p}\right)$ there exists a $t_{e}=t_{e}\left(u_{0}\right)$ such that the evolution equation (1.1) given on $\mathscr{X}$ has a unique (classical) solution $u$ on the time interval $\left[0, t_{e}\right]$, and $u \in C\left(\left[0, t_{e}\right], \mathscr{D}\left(A^{p}\right)\right)$. The proofs can be found in [15, Theorem 6.1.4; remark after Theorem 6.1.7]. The differentiability of $g$ on $\mathscr{X}$ yields 
that the related nonlinear flow $\psi_{t} u_{0}$ is well defined for every $u_{0} \in \mathscr{X}$ on sufficiently short time intervals. Furthermore, if $\psi_{[-t, t]} v, \psi_{[-t, t]} w \subseteq \mathscr{B}_{r}$ then

$$
\left\|\psi_{t} v-\psi_{t} w\right\| \leq \mathrm{e}^{|t| L_{r}}\|v-w\|
$$

where $L_{r}$ denotes the Lipschitz constant of $g$ on $\mathscr{B}_{r}$.

Next, consider the quadrature errors $R_{\ell}$ with $\ell=1, \ldots, p$, introduced in (2.1). The quadrature rule $Q_{\ell}$ is required to be of order $(p-\ell+1)$ and the integrands of $I_{\ell}$ are made up by compositions of the semigroup $\mathrm{e}^{t A}$ and the derivatives $g^{(k)}, 0 \leq k \leq \ell-1$. The integrands of $R_{\ell}$ are therefore obtained by differentiating the $I_{\ell}$ integrands $(p-$ $\ell+1)$ times. Observe that each of these differentiations generates exactly one $A$ term in the integrand. The assumed continuity properties of $g^{(k)}$ and the exact solution $u(t)$ then yield that the integrands of $R_{\ell}$ applied to $u(t)$ are all continuous in $\mathscr{X}$ with respect to $\left(t, \tau_{k}\right)$. Here, $\tau_{k}$ denotes the time dependences introduced by the $\mathrm{e}^{t A}$ terms in the integrands. Hence, $R_{\ell}$ evaluated at $u(t)$ is uniformly bounded in $\mathscr{X}$ with respect to $t \in\left[0, t_{e}\right]$. The same holds true for the term $R_{p+1}^{R}$ if $\tau$ is chosen small enough.

Bounding the global error $e_{n}=u(n \tau)-S^{n} u_{0}$ now follows in a standard fashion: choose $\mathscr{B}_{r^{\prime \prime}} \subset \mathscr{B}_{r^{\prime}} \subset \mathscr{B}_{r}$ such that $u(t) \in \mathscr{B}_{r^{\prime \prime}}$ for all $t \in\left[0, t_{e}\right]$ and $\tau$ sufficiently small such that $S v \in \mathscr{B}_{r}$ for all $v \in \mathscr{B}_{r^{\prime}}$. Assume that $S^{k} u_{0} \in \mathscr{B}_{r^{\prime}}$ for $k=0, \ldots, n$, then

$$
\begin{aligned}
\left\|e_{n+1}\right\| & \leq\left\|\sum_{\ell=1}^{p}\left(I_{\ell}-\tau^{\ell} Q_{\ell}\right) u\left(t_{n}\right)+\tau^{p+1} R_{p+1}^{R}\right\|+\left\|S u\left(t_{n}\right)-S^{n+1} u_{0}\right\| \\
& \leq \tau^{p+1}\left(\sum_{\ell=1}^{p}\left\|R_{\ell}\right\|+\left\|R_{p+1}^{R}\right\|\right)+\mathrm{e}^{\tau|\beta| L_{r}}\left\|e_{n}\right\|,
\end{aligned}
$$

where $t_{n}=n \tau$ and $|\beta|=\left|\beta_{1}\right|+\ldots+\left|\beta_{q-1}\right|$. The above recursion yields the bound

$$
\left\|e_{n+1}\right\| \leq \tau^{p} t_{e} \mathrm{e}^{t_{e}|\beta| L_{r}} \sup _{t \in\left[0, t_{e}\right]}\left(\sum_{\ell=1}^{p}\left\|R_{\ell}\right\|+\left\|R_{p+1}^{R}\right\|\right) .
$$

For $\tau$ chosen sufficiently small the bound implies that $S^{n+1} u_{0} \in \mathscr{B}_{r^{\prime}}$, and by induction we obtain the following convergence result:

Theorem 6.3 Consider the semilinear evolution equation (1.1) with solution $u$ and its approximation by a exponential splitting scheme $S$ given in (1.2). If Assumptions 6.1 and 6.2 hold, $S$ satisfies the order conditions $\{c(i, j)\}$ with $i \leq p \leq 4$ in Table 5.1, $u_{0} \in \mathscr{D}\left(A^{p}\right)$, and $\tau$ is sufficiently small, then

$$
\left\|u(n \tau)-S^{n} u_{0}\right\| \leq C \tau^{p}, \quad 0 \leq n \tau \leq t_{e},
$$

where the constant $C$ is independent of $n$ and $\tau$ separately.

Example 6.4 In order to illustrate the proposed assumptions on the vector field $A+g$, we once more consider the nonlinear wave equation (1.3). The first-order form of the equation equipped with periodic boundary conditions yields a semilinear evolution equation (1.1) on $\mathscr{X}_{m}=H^{m+1}\left(\mathbb{T}^{d}\right) \times H^{m}\left(\mathbb{T}^{d}\right)$. Here, $\mathbb{T}^{d}$ denotes a $d$-dimensional torus and the nonnegative integer $m$ satisfies $m>d / 2-1$. The latter implies that the Sobolev space $H^{m+1}\left(\mathbb{T}^{d}\right) \subset C\left(\mathbb{T}^{d}\right)$ is an algebra. The operator $A$, with $\mathscr{D}\left(A^{k}\right)=$ 
$\mathscr{X}_{m+k}$, generates a $C_{0}$ group and satisfies Assumption 6.1 (case a); compare with [15, Section 7.4].

Furthermore, if $\ell>d / 2$ and the function $f: \mathbb{R} \rightarrow \mathbb{R}$ is in $C^{\ell+k}(\mathbb{R})$, then the corresponding map $f: H^{\ell}\left(\mathbb{T}^{d}\right) \rightarrow H^{\ell}\left(\mathbb{T}^{d}\right)$, defined as $f(u)(x)=f(u(x))$, is $k$-times continuously Fréchet differentiable; compare with the proof of [16, Lemma 10.7]. Hence, the vector field $g(v, w)=(0, f(v))$ satisfies Assumption 6.2 if $f \in C^{m+p+2}(\mathbb{R})$.

\section{Schemes with approximated nonlinear flows}

As in the case of the introductory example, the action of $\mathrm{e}^{t A}$ can usually be computed in a very efficient manner, e.g., by Krylov methods or FFT. This is most commonly not the case for the nonlinear flow $\psi_{t}$. We therefore turn our attention to splitting schemes of the form

$$
T=\mathrm{e}^{\alpha_{q} \tau A} \varphi_{\beta_{q-1} \tau} \mathrm{e}^{\alpha_{q-1} \tau A} \ldots \varphi_{\beta_{1} \tau} \mathrm{e}^{\alpha_{1} \tau A}
$$

where $\varphi_{t}$ is a one-step approximation of $\psi_{t}$ which is assumed to fulfill the following:

Assumption 7.1 The map $\varphi_{t}$ satisfies a bound of the form (6.1) and is an order $p$ approximation of $\psi_{t}$, i.e., for $u_{0} \in \mathscr{X}$ and sufficiently small values of $\tau$ one has that

$$
\varphi_{\tau} u_{0}-\psi_{\tau} u_{0}=\tau^{p+1} P_{p} u_{0}
$$

where $P_{p}$ is a linear combination of compositions consisting of $\psi_{t}$ and the first $p$ derivatives of the nonlinear vector field $g$.

A single step with the splitting scheme $T u_{0}$ is within an $\mathscr{O}\left(\tau^{p+1}\right)$ vicinity of the exponential scheme $S u_{0}$, as

$$
\begin{aligned}
\left\|S u_{0}-T u_{0}\right\| & \leq \sum_{\ell=1}^{q-1} \mathrm{e}^{\tau\left(\left|\beta_{\ell}\right|+\ldots+\left|\beta_{q-1}\right|\right) L_{r}}\left\|\left(\varphi_{\beta_{\ell} \tau}-\psi_{\beta_{\ell} \tau}\right) \mathrm{e}^{\alpha_{\ell} \tau A} \prod_{i=1}^{\ell-1} \psi_{\beta_{i} \tau} \mathrm{e}^{\alpha_{i} \tau A} u_{0}\right\| \\
& \leq C \tau^{p+1} \sum_{\ell=1}^{q-1}\left\|P_{p} \mathrm{e}^{\alpha_{\ell} \tau A} \prod_{i=1}^{\ell-1} \psi_{\beta_{i} \tau} \mathrm{e}^{\alpha_{i} \tau A} u_{0}\right\| .
\end{aligned}
$$

Combining this observation with the convergence analysis for the exponential scheme yields that the new splitting scheme is also convergent of order $p$. We collect this result in the corollary below.

Corollary 7.1 Consider the semilinear evolution equation (1.1) with solution $u$ and its approximation by the splitting scheme $T$ given in (7.1). If Assumptions 6.1, 6.2, and 7.1 hold, the underlying exponential splitting scheme $S$ satisfies the order conditions $\{c(i, j)\}$ with $i \leq p \leq 4$ in Table 5.1, $u_{0} \in \mathscr{D}\left(A^{p}\right)$, and $\tau$ is sufficiently small, then

$$
\left\|u(n \tau)-T^{n} u_{0}\right\| \leq C \tau^{p}, \quad 0 \leq n \tau \leq t_{e},
$$

where the constant $C$ is independent of $n$ and $\tau$ separately. 


\section{Conclusions}

We have derived necessary and sufficient stiff order conditions, up to order four, for exponential splitting schemes applied to semilinear evolution equations, including nonlinear wave equations and diffusion-reaction processes. By identifying the local splitting error as a sum of quadrature errors, the stiff order conditions can be obtained in an explicit fashion, similarly to that of Runge-Kutta schemes. In fact, the stiff order conditions coincide with the classical non-stiff order conditions. We have also utilized the stiff order concept in an abstract convergence analysis. The latter extends to the case where the nonlinear flows in the splitting scheme are approximated by a sufficiently accurate one-step method.

Hence, in stark contrast to Runge-Kutta schemes and exponential integrators, there does not exist any additional stiff order conditions for exponential splitting schemes in the current semilinear context nor in the setting of linear evolution equations governed by two unbounded vector fields [8]. Furthermore, the Lie calculus [6, Section III.5.3] yields that no extra order conditions are introduced when passing from linear to nonlinear (non-stiff) problems, and we therefore conclude by conjecturing that a similar Lie based analysis will give the result below.

Conjecture 8.1 An exponential splitting scheme applied to a nonlinear evolution equation $\dot{u}=(f+g)(u)$ retains its classical non-stiff convergence order when $f$ and $g$ are sufficiently smooth and compatible.

\section{References}

1. C. Besse, B. Bidégaray, and S. Descombes, Order estimates in time of splitting methods for the nonlinear Schrödinger equation, SIAM J. Numer. Anal., 40, 26-40 (2002).

2. S. Blanes, F. Casas, P. Chartier, and A. Murua, Optimized high-order splitting methods for some classes of parabolic equations, Math. Comp., 82, 1559-1576 (2013).

3. F. Castella, P. Chartier, S. Descombes, and G. Vilmart, Splitting methods with complex times for parabolic equations, BIT, 49, 487-508 (2009).

4. E. Faou, Analysis of splitting methods for reaction-diffusion problems using stochastic calculus, Math. Comp., 78, 1467-1483 (2009).

5. D. Goldman and T. Kaper, Nth-order operator splitting schemes and nonreversible systems, SIAM J. Numer. Anal., 33, 349-367 (1996).

6. E. Hairer, C. Lubich, and G. Wanner, Geometric Numerical Integration, Springer, Berlin, 2006.

7. E. Hansen, F. Kramer, and A. Ostermann, A second-order positivity preserving scheme for semilinear parabolic problems, Appl. Numer. Math., 62, 1428-1435 (2012).

8. E. Hansen and A. Ostermann, Exponential splitting for unbounded operators, Math. Comp., 78, 14851496 (2009).

9. E. Hansen and A. Ostermann, High order splitting methods for analytic semigroups exist, BIT, 49, 527-542 (2009).

10. H. Holden, C. Lubich, and N.H. Risebro, Operator splitting for partial differential equations with Burgers nonlinearity, Math. Comp., 82, 173-185 (2013).

11. W. Hundsdorfer and J. Verwer, Numerical solution of time-dependent advection-diffusion-reaction equations, Springer, Berlin, 2003.

12. T. Jahnke and C. Lubich, Error bounds for exponential operator splittings, BIT, 40, 735-744 (2000).

13. C. Lubich, On splitting methods for Schrödinger-Poisson and cubic nonlinear Schrödinger equations, Math. Comp., 77, 2141-2153 (2008).

14. A. Murua and J. Sanz-Serna, Order conditions for numerical integrators obtained by composing simpler integrators, Phil. Trans. R. Soc. Lond. A, 357, 1079-1100 (1999). 
15. A. Pazy, Semigroups of Linear Operators and Applications to Partial Differential Equations, Springer, New York, 1983

16. M. Renardy and R. Rogers, An Introduction to Partial Differential Equations, Springer, New York, 2004.

17. M. Suzuki, Fractal decomposition of exponential operators with applications to many-body theories and Monte Carlo simulations, Phys. Lett. A, 146, 319-323 (1990).

18. R. Temam, Infinite-Dimensional Dynamical Systems in Mechanics and Physics, Springer, New York, 1997.

19. M. Thalhammer, High-order exponential operator splitting methods for time-dependent Schrödinger equations, SIAM J. Numer. Anal., 46, 2022-2038 (2008). 